40.

\title{
ON THE THEORY OF INVOLUTION IN GEOMETRY.
}

[From the Cambridge and Dublin Mathematical Journal, vol. Il. (1847), pp. 52-61.]

WHEN three conics have the same points of intersection, any transversal intersects the system in six points, which are said to be in involution. It appears natural to apply the term to the conics themselves; and then it is easy to generalize the notion of involution so as to apply it to functions of any number of variables. Thus, if $U, V$... be homogeneous functions of the same order of any number of variables $x, y \ldots$, a function $\Theta$, which is a linear function of $U, V \ldots$, is said to be in involution with these functions. More generally $\Theta$ may be said to be in involution with any system of factors of these functions: or if $U, V \ldots$ be given functions of $x, y, z \ldots$, homogeneous of the degrees $m, n \ldots$, and $u, v, \ldots$ arbitrary homogeneous functions of the degrees $r-m, r-n \ldots$; then, if $\Theta=u U+v V+\ldots, \Theta$ is a function of the degree $r$, which is in involution with $U, V \ldots$. The question which immediately arises, is to find the degree of generality of $\Theta$, or the number of arbitrary constants which it contains. And this is a question which, from the variety and interest of its geometrical interpretations, has very frequently been treated of by geometers, though never, I believe, in quite so general a form, (the number $r$ has almost always had particular values given to it, except in a short paper of my own, on the particular case of two curves, in the Journal, vol. III. p. 211 [5]). ${ }^{1}$ There is also an analytical application of

1 The first suggestion of the problem is contained in a memoir of Euler's-" Sur une contradiction apparente dans la doctrine des lignes courbes," Mém. de Berlin, t. Iv. [1748] p. 219. It is noticed also in Cramer's Introduction à l'analyse des lignes courbes [1750]. The following memoirs also have been published on the subject: Plücker, "Recherches sur les courbes algébriques de tous les degrés," Gerg. Ann. t. xIx. [1828-29] p. 97; "Recherches sur les surfaces algébriques de tous les degrés," p. 129; (a great number of memoirs on particular applications of the theory are contained in Gergonne;) Jacobi, "De relationibus quæ locum habere debent inter puncta intersectionis duarum curvarum vel trium superficierum dati ordinis, simul cum enodatione paradoxi algebraici," Crelle, t. xv. [1836]; Plücker, "Théorèmes généraux concernant les équations d'un degré quelconque entre un nombre quelconque d'inconnues," Crelle, t. xvi. [1837], (but this last must be read with caution, as several of the theorems are incorrect, or at least stated without the proper limitations); and the Einleitende Betrachtungen, in Plücker's "Theorie der algebraischen Curven" [1839]. The following memoirs of Hesse, containing developments relative to the case of three surfaces of the second order, may likewise be mentioned, "De curvis et superficiebus secundi gradus," Crelle, t. xx. [1840] p. 285; and "Ueber die lineare Construction des achten Schnitt-punctes dreier Oberflächen zweiter Ordnung, wenn sieben Schnitt-puncte derselben gegeben sind," Crelle, t. xxvr. [1843] p. 147. 
the theory, of considerable interest, to the problem of elimination between any number of equations containing the same number of variables. Suppose, for instance, two equations, $U=0, V=0$, when $U, V$ are homogeneous functions of $x, y$ of the degrees $m, n$ respectively. To eliminate the variables it is sufficient to multiply the first equation by $x^{n-1}, x^{n-2} y \ldots, y^{n-1}$, and the second by $x^{m-1} \ldots, y^{m-1}$, and from the equations so obtained to eliminate linearly the $(m+n)$ quantities $x^{m+n-1}, x^{m+n-2} y \ldots, y^{m+n-1}$. But in the case of a greater number of equations it is not at first obvious how many new equations should be obtained; and when a number apparently sufficiently great have been found, it may happen that the equations so obtained are not independent, and that the elimination cannot be performed. But in showing the connexion that exists between these different equations, the theory of involution explains in what manner a system is to be formed, which includes all the really independent equations, and gives the means of detecting the extraneous factors which appear in the result of the linear elimination of the different terms; but I do not see at present any mode of obtaining the final result at once in its reduced form free from any extraneous factors.

Let $X, Y, \ldots$ be given homogeneous functions of the same degree of any number of variables, and suppose

$$
\Theta=\alpha X+\beta Y+\ldots,
$$

$\alpha, \beta \ldots$ being constants, and the number of terms in the series being $g ; \Theta$ contains therefore $g$ arbitrary constants. If however, by giving to $\alpha, \beta \ldots$ particular values $\alpha_{1}, \beta_{1} \ldots$, or $\alpha_{2}, \beta_{2} \ldots$, and representing by $\Theta_{1}, \Theta_{2} \ldots$ the corresponding values of $\Theta$, we have identically

$$
\Theta_{1}=0, \quad \Theta_{2}=0, \ldots(h \text { equations }) ;
$$

then the constants in $\Theta$ group themselves together into a smaller number $g-h$ of arbitrary constants. This supposes, however, that the last mentioned equations are linearly independent; if there are a certain number $k$ of equations

$$
\Phi_{1}=0, \quad \Phi_{2}=0 \ldots,
$$

(where $\Phi_{1}, \Phi_{2}, \ldots$ are linear functions of $\Theta_{1}, \Theta_{2} \ldots$ ) which are identically satisfied, independently of the $h$ equations, then the equations in question are equivalent to $h-k$ equations, and the function $\Theta$ contains $g-(h-k)$ or $g-h+k$, arbitrary constants. Similarly if the functions $\Phi$ are not independent; so that the number of arbitrary constants really contained in $\Theta$ is always

$$
N=g-h+k-\& c . . .
$$

Consider now the case of a function $\Theta$, homogeneous of the $r^{\text {th }}$ degree in the variables $x, y \ldots\{(\theta+1)$ in number $\}$. Let $U, V \ldots$ be functions of the degrees $m, n \ldots$, and suppose

$$
\Theta=u U+v V+\ldots
$$

where $u, v \ldots$ are arbitrary functions of the degrees $r-m, r-n, \ldots\{r$ is supposed throughout greater than $m, n \ldots\}$. Suppose for shortness that the number of terms in the complete function of $\theta$ variables, and of the order $\rho$, i.e. the quotient $\frac{[\rho+\theta]^{\theta}}{[\theta]^{\theta}}$, is 
represented by $[\rho, \theta]$; then the function $\Theta$ contains apparently a number

of arbitrary constants.

$$
([r-m, \theta]+[r-n, \theta]+\ldots)
$$

But since we should have identically $\Theta=0$ by assuming $u=L V, v=-L U, w=0, \& \mathrm{c} \ldots$. ( $L$ the general function of the order $r-m-n$ ), or $u=M W, v=0, w=-M U$ ( $M$ the general function of the order $r-m-p$ ) \&c., the number $N$ must be diminished by

$$
[r-m-n, \theta]+[r-m-p, \theta]+[r-n-p, \theta]+\ldots ;
$$

but the equations just obtained are themselves not linearly independent, and in consequence of this the number of arbitrary constants has to be increased by

$$
[r-m-n-p, \theta]+\ldots ;
$$

and so on. Hence finally the whole number of arbitrary constants in the function $\theta$ is

$$
\begin{gathered}
N=[r-m, \theta]+[r-n, \theta]+[r-p, \theta]+\ldots \\
-[r-m-n, \theta]-[r-m-p, \theta]-[r-n-p, \theta]-\ldots \\
+[r-m-n-p, \theta]+\ldots \pm \& \text { \&. \&c. }
\end{gathered}
$$

This however supposes that all the numbers $r-m, r-n \ldots, r-m-n \ldots$, are positive : whenever this is not the case for any one of them, the corresponding term is obviously to be omitted. With this convention the equation (A) gives always the correct number of arbitrary constants in $\Theta$ : it will be convenient to represent it in the abbreviated form

$$
N=\{r: m, n, p, \ldots: \theta\} .
$$

An expression analogous to this, for the particular case of $r=m$, but incorrect on account of the omission of all the terms after the second line, has been given by M. Plücker (Crelle, tom. xvI. p. 55), and even some of his particular formulie are incorrect. But proceeding to examine some particular cases: if $r>m+n+p+\ldots-\theta-1$, then in the expression (A) either no terms are to be omitted, or else the terms to be omitted reduce themselves to zero, so that $N$ is given by this formula continued to its last term. It will be subsequently shown that in this case

$$
\{r: m, n, p \ldots: \theta\}=[r, \theta]-m n p \ldots ;
$$

or in the case of two or three variables, we have the theorem, "If a curve or surface of the order $r$ be determined to pass through the $m n$ points of intersection of two curves of the orders $m$ and $n$, or the $m n p$ points of intersection of three surfaces of the orders $m, n, p$; then if $r>m+n-3$, or $r>m+n+p-4$, the curve or surface contains precisely the same number of arbitrary constants as if the $m n$ or $m n p$ points were perfectly arbitrary."

This is natural enough; the peculiarity is in the case where $r \ngtr m+n-3$, or $r \ngtr m+n+p-4$. For instance, for two curves, $r \ngtr m+n-3$, we have

$$
\{r: m, n: 2\}=[r-m, 2]+[r-n, 2]=[r, 2]-m n+[r-m-n, 2] \text {, }
$$


or the new curve contains $\frac{1}{2}[m+n-r-1]^{2}$ more arbitrary constants than it would do if the $m n$ points, through which it was made to pass, had been perfectly arbitrary; a result given before in the Journal, [5].

In the case of surfaces, if $r \ngtr m+n+p-4$. Then assuming $r>m+n-4, m+p-4$, or $n+p-4$, we have

$$
\begin{aligned}
\{r: m, n, p: 3\} & =[r-m, 3]+[r-n, 3]+[r-p, 3] \\
& -[r-m-n, 3]-[r-m-p, 3]-[r-n-p, 3] \\
& =[r, 3]-m n p-[r-m-n-p, 3] ;
\end{aligned}
$$

or the surface contains $\frac{1}{6}[m+n+p-r-1]^{3}$ more arbitrary constants than it would do if the mnp points, through which it was made to pass, had been perfectly arbitrary. Similarly, in the case where $r$ is not greater than one or more of the quantities $m+n-4, m+p-4, n+p-4$. Thus in particular, if $r$ be not greater than the least of these quantities

$$
\begin{aligned}
\{r: m, n, p: 3\}=[r, 3]-m n p & +[r-n-p, 3]+[r-m-p, 3] \\
+ & {[r-m-n, 3]-[r-m-n-p, 3] }
\end{aligned}
$$

or the surface contains

$$
\frac{1}{6}[m+n+p-r-1]^{3}-\frac{1}{6}[n+p-r-1]^{3}-\frac{1}{6}[m+p-r-1]^{3}-\frac{1}{6}[m+n-r-1]^{3}
$$

more arbitrary constants than it would otherwise have done. Again, for a surface of the $r^{\text {th }}$ order, subjected to pass through the curve of intersection of two surfaces of the orders $m, n$,

$$
\{r: m, n, 3\}=[r-m, 3]+[r-n, 3]-[r-m-n, 3] ;
$$

in which the last term, or $\frac{1}{6}[m+n-r-1]^{3}$, is to be omitted when $r \ngtr m+n-4$.

The function of the $r^{\text {th }}$ order, which is satisfied by the systems of values which satisfy the equations of the orders $m, n \ldots$ contains, we have seen, $[r, m, n, p \ldots \theta]$ arbitrary constants; hence it may be determined so as to pass through this number, diminished by unity, of arbitrary points. But the equation being determined in general by the condition of being satisfied by $[r, \theta]-1$ systems of variables, it will be completely determined if, in addition to the above number of arbitrary systems, we suppose it to be satisfied by a number $N=[r, \theta]-\{r ; m, n, p \ldots: \theta\}$ of systems satisfying the equations above. Hence the theorem

"The equation of the $r^{\text {th }}$ order which is satisfied by a number

$$
N=[r, \theta]-\{r ; m, n, p \ldots: \theta\}
$$

of systems satisfying the equations of the orders $m, n, p \ldots$ is satisfied by any systems whatever which satisfy these equations."

In particular- "The surface of the $r^{\text {th }}$ order which passes through a number

$$
[r, \theta]-\{r: m, n: \theta\}
$$


of points in the curve of intersection of two surfaces of the orders $m, n,-$ or through $[r, \theta]-\{r: m, n, p: \theta\}$ of the $m n p$ points of intersection of three surfaces of the orders $m, n, p$,-passes through the curve of intersection, or through the mnp points of intersection."

Thus a surface of the second order which passes through eight points of the curve of intersection of two surfaces of the second order passes through this curve; and any surface of the second order which passes through seven of the points of intersection of three surfaces of the second order passes through the eighth point. (The first theorem obviously fails if the eight points have the relation in question, i.e. if they are the eight points of intersection of three surfaces of the second order.)

Again-" The curve of the $r^{\text {th }}$ order which passes through $[r, \theta]-\{r: m, n: \theta\}$ of the points of intersection of two curves of the orders $m n$, passes through the remaining points of intersection." e.g. "Any curve of the third order which passes through eight of the points of intersection of two curves of the third order, passes also through the ninth point."

Consider next the following question, which [as regards particular cases] has been treated of by Jacobi in the memoir already quoted (Crelle, tom. xv.). "To find the number of relations which must exist between $K(\theta+1)$ variables, forming $K$ systems, each of which satisfies simultaneously equations of the orders $m, n, p \ldots$ respectively; the number $\phi$ of these equations being anything less than $\theta$; or $\phi$ being equal to $\theta$, provided at the same time $K=m n p \ldots . . "$

Suppose $m \nless n, n \nless p \ldots$ and write

$$
\begin{aligned}
& {[m, \theta]-\{m: m, n, p \ldots: \theta\}=N,} \\
& {[n, \theta]-\{n: n, p \ldots \ldots .: \theta\}=N^{\prime},} \\
& \& c .
\end{aligned}
$$

Imagine the equations of the orders $n, p \ldots$ given. Any function of the $m^{\text {th }}$ order which is satisfied by $N$ of the systems of values which satisfy the given equations, and any particular equation of the $m^{\text {th }}$ order, is satisfied by the remaining $K-N$ systems of values. Hence assuming $N$ systems, satisfying the equations of the orders $n, p \ldots$ but otherwise arbitrary, the remaining systems must satisfy these equations, and a completely determinate equation of the $m^{\text {th }}$ order; i.e. there must be $\phi$ relations between the variabless of each system, and consequently $\phi(K-N)$ relations in all. Similarly, if the equations of the orders $p \ldots$ were given, $N^{\prime}$ systems of variables might be assumed satisfying these equations, but otherwise arbitrary; the remaining $N-N^{\prime}$ systems satisfy $(\phi-1)$ determinate equations, or the number of relations between the variables is $(\phi-1)\left(N-N^{\prime}\right) \ldots$; continuing in the same manner the total number of relations between the variables is

$$
\phi(K-N)+(\phi-1)\left(N-N^{\prime}\right)+(\phi-2)\left(N^{\prime}-N^{\prime \prime}\right)+\ldots
$$

in which however any term $(\phi-1)\left(N-N^{\prime}\right)$ or $(\phi-2)\left(N-N^{\prime}\right) \ldots$ \&c., which becomes negative, must be omitted. It is obvious that we may write more simply

$$
\begin{aligned}
& N=[m, \theta]-1-\{m ; n, p \ldots \theta\}, \\
& N^{\prime}=[n, \theta]-1-\{n ; p \ldots \theta\}, \& c .
\end{aligned}
$$


In particular, to find the relations which must exist between the coordinates of $m n$ points in order that they may be the points of intersection of two curves of the orders $m, n$ respectively: here $K=m n, N=\frac{1}{2}[m+2]^{2}-\frac{1}{2}[m-n+2]^{2}=\frac{1}{2}\left(2 m n-n^{2}+3 n\right)$, $N^{\prime}=\frac{1}{2}\left(n^{2}+3 n+2\right)$, so that $N-N^{\prime}=m(m-n)-1$ which becomes negative when $m=n$; hence in general the required number of conditions is $m n-3 n+1$, but when $m=n$. the number in question becomes $(n-1)(n-2)$.

Passing to the case of surfaces; to determine the number of relations which must exist between the coordinates of mnp points, in order that they may be the points of intersection of surfaces of the orders $m, n, p$ respectively. The number required is

$$
3(m n p-N)+2\left(N-N^{\prime}\right)+\left(N^{\prime}-N^{\prime \prime}\right),
$$

where

$$
N=[m, 3]-1-[m-n, 3]-[m-p, 3]+[m-n-p, 3]
$$

(this last term to be omitted when $m<n+p-3$ ),

$$
\begin{aligned}
& N^{\prime}=[n, 3]-1-[n-p, 3], \\
& N^{\prime \prime}=[p, 3]-1 .
\end{aligned}
$$

If, for instance, $m>n+p-3$, so as to retain the term [ $m-n-p, 3]$, and $n>p$, so as to retain the term $N^{\prime}-N^{\prime \prime}$, the number becomes, after all reductions,

$$
2 m n p+n p^{2}-4 n p-2 p^{2}-\frac{1}{3}(p-1)(p-2)(p-3),
$$

a formula given by Jacobi. If, however, $n=p$, this number must be augmented by unity. Again, for $m<n+p-3$, the required number is

$$
\begin{aligned}
2 m n p+n p^{2}- & 4 n p-2 p^{2}-\frac{1}{3}(p-1)(p-2)(p-3) \\
& -\frac{1}{6}(n+p-m-1)(n+p-m-2)(n+p-m-3),
\end{aligned}
$$

which however must be augmented by unity if $m=n$ or $n=p$, and by 3 if $m=n=p$. But without entering into further details about this part of the subject, which has been sufficiently illustrated by the examples that have been given, I pass on to notice the application of the above theory to the problem of elimination. Imagine $(\theta+1)$ equations between the $(\theta+1)$ variables, the first sides of these being, as before, rational and integral homogeneous functions of the variables of the orders $m, n, p \ldots$ respectively. Writing $m+n+p \ldots-\theta=r$, and multiplying the first equation by all the terms of the form $x^{a} y^{\beta} \ldots$ of the degree $r-m$, the second equation by all the terms of the same form, of the degree $r-n$, and so on, there result a certain number of equations, containing all the terms $x^{a} y^{\beta} \ldots$ of the degree $r$. But these equations are not independent; and the reasoning in the former part of the present paper shows that the number of independent equations is given by the symbol $\{r: m, n, p \ldots: \theta\}$; the number of terms $x^{a} y^{\beta} \ldots$ is evidently $[r, \theta]$; and it will be shown immediately that for the actual value of $r$,

$$
[r, \theta]-\{r ; m, n, p \ldots: \theta\}=0
$$

so that the number of quantities to be linearly eliminated is precisely equal to the number of equations, or the elimination is always possible. I may mention also that, 
supposing the coefficients of all the equations to be of the order unity, the order of the result, free from extraneous factors, may be shown to be

$$
\begin{array}{r}
{[r-m, \theta]+\ldots-2\{[r-m-n, \theta]+\ldots\}+3\{[r-m-n-p, \theta]+\ldots\}-\& c .} \\
=m n \ldots+m p \ldots+n p \ldots+\& c . \ldots \ldots \ldots \ldots \ldots \ldots \ldots \ldots
\end{array}
$$

(the equality of which will be presently proved) a result which agrees with that deduced from the theory of symmetrical functions; but I am not in possession of any mode of directly obtaining the final result in this its most simplified form. My method, which it is not necessary to explain here more particularly, leads me to the formation of a set of functions

$$
P, Q, \ldots \ldots X, Y, Z,
$$

$\theta$ in number, such that $Z$ divides $Y$, this quotient divides $X$, and so on until we have a certain quotient which divides $P$, and this quotient equated to zero is the result of the elimination freed from extraneous factors. It only remains to demonstrate the formulæ (A), (B), and (C). Suppose in general that (k) denotes the sum of all the terms of the form $m^{a} n^{b} \ldots$, which can be formed with a given combination of $k$ letters out of the $\phi$ letters $m, n, p \ldots$; and let $\Sigma(k)$ denote the sum of all the series $(k)$ obtained by taking all the possible different combinations of $k$ letters. It is evident that $\Sigma^{\prime}(k)$ is a multiple of $(\phi),\{(\phi)$ denoting of course the sum of all the terms. $m^{a} n^{b} \ldots, m, n \ldots$ being any letters whatever out of the series $\left.m, n, p \ldots\right\}$. Let $g$ be the number of exponents $a, b, \ldots$, then $(\phi)$ contains $[\phi]^{g}$ terms, also $(k)$ contains $[k]^{g}$ terms, and the number of terms such as $(k)$ in the sum $\Sigma(k)$ is $[\phi]^{\phi-k} \div[\phi-k]^{\phi-k}$. Hence evidently

$$
\Sigma(k)=\frac{[\phi-g]^{\phi-k}}{[\phi-k]^{\phi-k}}(\phi),
$$

or, what comes to the same thing,

$$
\Sigma(\phi-k)=\frac{[\phi-g]^{k}}{[k]^{k}}(\phi) .
$$

Let $A$ be an indeterminate coefficient, $\sigma$ a summatory sign referring to different systems of exponents; then

$$
\Sigma \sigma A(\phi-k)=\sigma \frac{[\phi-g]^{k}}{[k]^{k}} A(\phi),
$$

or, giving to $k$ the values $1,2 \ldots \phi$, multiplying each equation by an arbitrary coefficient, and adding, putting also for shortness $\sigma A(\phi-k)=U_{\phi-k}$, we have

$$
\alpha_{\phi} U_{\phi}+\alpha_{\phi-1} \Sigma U_{\phi-1}+\ldots=\sigma\left(\alpha_{\phi}+\alpha_{\phi-1} \frac{[\phi-g]^{1}}{[1]^{1}}+\ldots\right) A(\phi)
$$

whence in particular,

$$
\begin{aligned}
U_{\phi}-\Sigma U_{\phi-1}+\ldots & =\sigma\left\{0^{\phi-g} A(\phi)\right\}, \\
\Sigma U_{\phi-1}-2 \Sigma U_{\phi-2}+\ldots & =\sigma\left\{(\phi-g) 0^{\phi-g-1} A(\phi)\right\},
\end{aligned}
$$

C. 
which are still equations of considerable generality. If now $\phi=\theta$ and $U_{\theta}$ is a function of $m+n+p+\ldots$ of the order $\theta$, the quantity $\sigma\left\{0^{\theta-g} A(\theta)\right\}$ reduces itself to the single term of $U_{\theta}$ which contains the product mnp... Hence, if

$$
U_{\theta}=[\alpha+m+n+p \ldots, \theta]
$$

in which afterwards $\alpha=r-m-n-p-\ldots$ we have the formula (A). Again, if $\phi=\theta+1$, and $U_{\theta+1}$ is a function of $m+n+p \ldots$ of the order $\theta$, the sum $\sigma\left\{0^{\theta+1-g} A(\phi)\right\}$ vanishes; whence writing $U_{\theta+1}=[m+n+p \ldots-\theta, \theta]$, we have the formula (B). Similarly, if in the second formula $\phi=\theta+1$, and $U_{\theta+1}$ is a function of $m+n+p \ldots$ of the degree $\theta$, then

$$
\sigma\left\{(\theta+1-g) 0^{\theta-g} A(\theta+1)\right\},
$$

reduces itself to the term which contains $m n \ldots+n p \ldots+m p \ldots+\& c$; whence, if

$$
U_{\theta+1}=[m+n+p+\ldots-\theta, \theta],
$$

we have the formula (C). 